\title{
FIXTURELESS PROFILE INSPECTION OF NON-RIGID PARTS USING THE NUMERICAL INSPECTION FIXTURE WITH IMPROVED DEFINITION OF DISPLACEMENT BOUNDARY CONDITIONS
}

\author{
V. Sabri ${ }^{*}$, S.A. Tahan ${ }^{1}$, X.T. Pham ${ }^{1}$, D. Moreau ${ }^{2}$, and S. Galibois ${ }^{3}$ \\ vahid.sabri.1@ens.etsmtl.ca
}

\begin{abstract}
Quality control is an important factor for manufacturing companies looking to prosper in an era of globalization, market pressures, and technological advance. The functionality and product quality cannot be guaranteed without this important aspect. Manufactured parts have deviations from their nominal (CAD) shape caused by the manufacturing process. Thus, geometric inspection is a very important element in the quality control of mechanical parts. We have focused here on the profile inspection of non-rigid parts which are widely used in the aeronautic and automotive industries. Non-rigid parts can have different forms in a freestate condition compared with their nominal models due to residual stress and gravity loads. To solve this problem, dedicated inspection fixtures are generally used in industry to compensate for the displacement of such parts for simulating the use state in order to perform geometric inspections. These fixtures and the inspection process are expensive and time-consuming. Our aim is therefore to develop an inspection method which eliminates the need for specialized fixtures by acquiring a point cloud from the displaced part using a contactless measuring system such as optical scanning and comparing it with the CAD model for the identification of deviations. Using a non-rigid registration method and finite element analysis, we will numerically inspect the profile of a non-rigid part. To do so, a simulated displacement is performed using an improved definition of boundary conditions for simulating unfixed parts. In this paper, we will apply an improved method on two industrial non-rigid parts with free-form surfaces simulated with different types of displacement, defect, and measurement noise.
\end{abstract}

Keywords: quality control, geometric inspection, geometric dimensioning and tolerancing, profile tolerance, registration, non-rigid/flexible/deformable part, assembly conditions, metrology.

\footnotetext{
* Corresponding Author
}

1. Department of Mechanical Engineering, École de Technologie Supérieure (ÉTS), Montreal, Canada

2. Bombardier Aerospace Inc., Montreal, QC, Canada

3. Creaform Inc., Levis, QC, Canada

The final publication is available at Springer via http://doi.org/10.1007/s00170-015-7425-3 


\section{INTRODUCTION}

Geometric inspection has an important role to play in the quality control of mechanical parts since it usually consumes a large portion of production lead time. By means of Geometric Dimensioning and Tolerancing (GD\&T), geometric specifications and product design are specified according to functionality. To verify whether manufactured parts meet specifications defined at the design phase, the GD\&T inspection process is applied. By using a reliable, efficient, and automated inspection process, product life cycle time will decrease and industrial competition will improve [1]. Although the methods for geometric inspection of rigid parts have significantly improved and are generally available within the industry [2], the geometric inspection of non-rigid parts with free-form surfaces has not been well studied.

In mechanical engineering applications, surfaces are allocated a profile tolerance to control manufacturing variations [2]. A surface profile should be controlled based on the principles established by the ASME Y14.5-2009 standards (section 8) [3]. According to these standards (or ISO 1101:2004, ISO-GPS standards [4]), unless otherwise specified, all tolerances should be applied in a free-state condition. Exemptions are agreed to this rule for non-rigid parts. In these cases, non-rigid parts may deform significantly from their defined tolerances due to their weight (gravity), or the release of residual stresses resulting from manufacturing processes [3, 5].

Generally, to solve the above-mentioned problem, special inspection fixtures with complex setups are used within the industry to compensate for the displacements to simulate use state in order to perform geometric inspection. These dedicated fixtures are expensive, heavy, and complex (Figure 1). The process is extremely time-consuming which reduces competitiveness. The mentioned standards also allow for the application of reasonable load (not exceeding the load expected under normal assembly conditions) to displace non-rigid parts to conform to the specified tolerances. The solution is to develop an inspection technique which eliminates the need for specialized fixtures by acquiring a point cloud from the displaced part using a contactless measuring system such as optical scanning and comparing it with the CAD model for the identification of deviations.
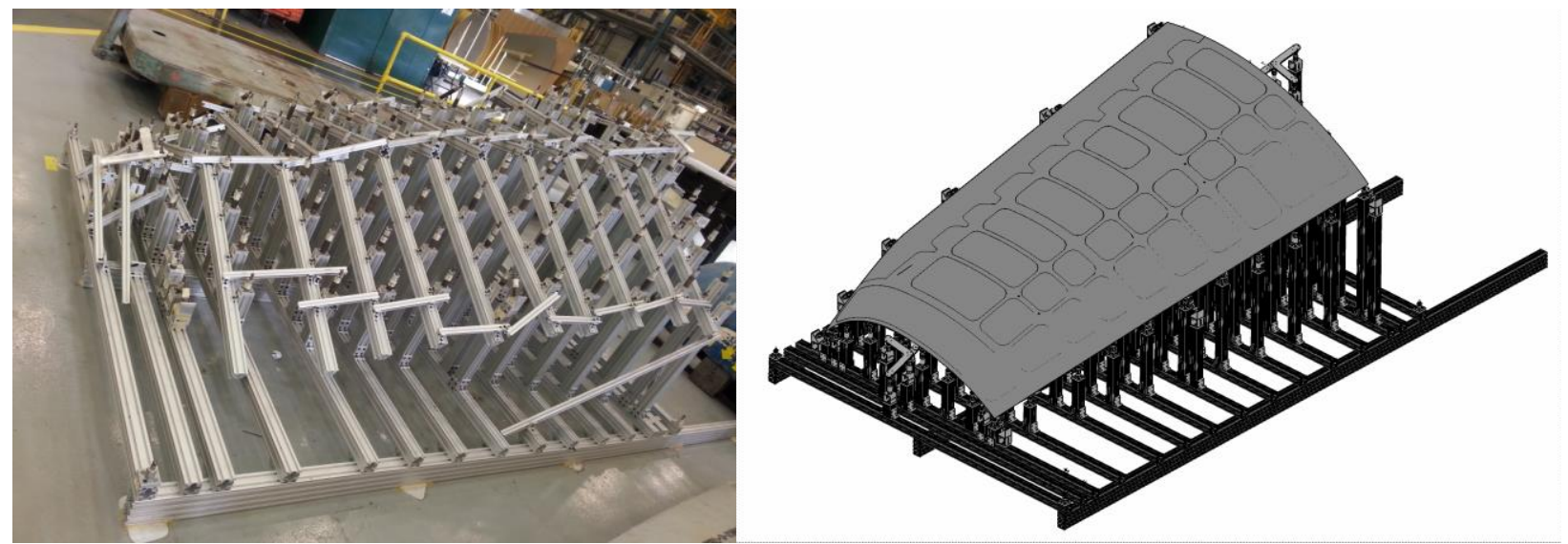

Figure 1: A special, expensive, heavy, and complex fixture for the inspection of a flexible plate, Bombardier Aerospace Inc., left: the fixture without the part, right: the CAD model of the fixture with the part set up on it

For the purpose of comparing the measurement data (point cloud) with the nominal model, it is necessary to dispose these two sets in a joint coordinate system. This procedure is called registration. In recent and modern technologies, this registration is mathematically defined using the translation and the rotation of the Design Coordinate System (DCS) with respect to the Measurement Coordinate System (MCS). In application, registration can be done in two steps: searching for the corresponding relationship between scanned and nominal surfaces, and finding an optimal transformation matrix between the DCS and MCS. 
The rigid registration methods are only applied for rigid parts whose shapes are similar. Thus, they do not cover flexible parts in which the registration problem requires application of a kind of non-rigid registration method in addition to finding a rigid mapping. The difference between rigid and non-rigid registrations is that a non-rigid registration can align two different shapes (for example, a line with a curve) [6, 7]. Several rigid and non-rigid registration methods have been developed such as the Iterative Closest Point (ICP) algorithm [8] and its variants for rigid registration; the Multi-Dimensional Scaling (MDS) method [9], and the Coherent Point Drift (CPD) algorithm [10] for non-rigid registration applied in medical imaging, animation, etc. However, the situation for the registration of a non-rigid mechanical part is different due to the result of its compliance behavior.

Compliance behavior of a compliant (flexible) part is an essential issue to study while specifying tolerances and assessing the geometric and dimensional specifications for the part. This factor is a relative concept based on the relation between an imposed force and its persuaded displacement [11]. Based on the displacements of parts induced by a reasonable force $(50 \mathrm{~N})$ during inspection, the parts are considered rigid/non-rigid (flexible)/extremely non-rigid (see Table 1). Another method for quantifying flexibility of the mechanical part, from an industrial point of view, was proposed by Aidibe and Tahan [12]. Their quantifying method is based on the ratio between the maximum displacement induced by a certain force and the profile tolerance of the non-rigid part. Our research is done on typical non-rigid mechanical parts used in the aeronautic and automotive industries.

Table 1: Displacement of parts in each zone induced by a force during inspection and their compliance behavior

\begin{tabular}{|l|l|}
\hline$\delta /$ tol by a reasonable force during inspection $(\approx 50 \mathrm{~N})$ & Compliance behavior \\
\hline$\delta /$ tol $<5-10 \%$ & Rigid \\
\hline $\begin{array}{l}\delta / \text { tol }>5-10 \% \quad \text { (e.g. thin shell, skin in aeronautic and } \\
\text { automotive parts) }\end{array}$ & Non-rigid (flexible) \\
\hline $\begin{array}{l}\delta / \text { tol } \gg 10 \% \text { (the shape depends on the part's weight } \\
\text { and position, such as thin seals and paper) }\end{array}$ & Extremely non-rigid \\
\hline
\end{tabular}

The following paper includes four sections: a review of previous researches for the fixtureless geometric inspection of non-rigid parts, the developed method, case studies including the presentation of metrological performances of our method, and finally, a conclusion.

\section{REVIEW OF PREVIOUS RESEARCH}

Ascione and Polini [13] dealt with the free-form surface inspection of non-rigid parts using inspection fixtures combined with CMM. Abenhaim et al. [11] presented a review of the previous researches for the fixtureless inspection of non-rigid parts and proposed a classification of the specification methods used for the GD\&T of non-rigid parts under the ASME and ISO standards. In the following, we will introduce the primary methods, based on the simulated displacement approach, developed for the geometric inspection of non-rigid parts without the use of inspection fixtures.

For the first time in 2006, Weckenmann et al. [14, 15] made strides in the fixtureless inspection of non-rigid parts by proposing the virtual distortion compensation method in which they virtually displaced the distorted part into the nominal model by displacing the point cloud captured by a contactless scanning device. A triangle mesh of the surface from the point cloud was generated and transformed into a finite element analysable (FEA) model. Afterwards, the fixation process was simulated using information about the assembly features' deviation from the actual (measured) to the ideal (nominal) position. This method requires human intervention to recognize the correlation between some determined points 
and assembly conditions in order to define the boundary conditions of the FEA problem. Therefore, boundary conditions can be improved to simulate a real model of the fixation system. In addition, converting the point cloud into a FE model is a time-consuming process with many uncertainties. In 2007, Weckenmann et al. [16] improved the shortcomings of their previous work by displacing a CAD model towards the measurement data in the virtual reverse deformation method. They enforced the boundary conditions on the CAD model using the known position of the fixation points on the scanned part. Therefore, a preprocessing of the measurement data is not needed. Through this method, they decreased inspection time and obtained more precise results. FE simulation of the displacement boundary conditions on the geometrically ideal CAD model is clearly more accurate. However, this method still required human intervention to find the corresponding relationship between the CAD model and the measurement data. Moreover, the modeling of the boundary conditions in the FE dataset needs to be improved to simulate the unfixed part.

Similar to the virtual reverse deformation method, Jaramillo et al. [17, 18] proposed an approach which requires significantly less computing power, using the Radial Basis Functions (RBFs) to minimize the finite element mesh density required to correctly predict part behavior. Recently in [19], they improved their method by performing flexible part registration using only partial views from areas that have to be inspected. They applied an interpolation technique based on RBFs to estimate positions of the missing fixation points since the partially scanned data may not contain all of them.

Gentilini and Shimada [20] proposed a method for the shape inspection of a flexible assembly part by virtually mounting it into the assembly. First, the dense measured mesh is smoothed and reduced to become suitable for FEA. If not available, material properties are defined by a calibration process. Then, specific displacement boundary conditions are defined and applied for FE simulation of the assembly process. Once FEA is performed, quality inspection of the simulated post-assembly shape is done using visualization tools. In addition, the virtual post-assembly shape is compared with the real post-assembly shape for method accuracy validation. This method can predict the final assembled shape of a flexible part, but it has the shortcomings mentioned in the virtual distortion compensation method. The polygonal mesh data suffers from uncertainties, noise, and a high quantity of polygons; therefore, it needs post-processing steps, smoothing, and decimation.

Recently, Radvar-Esfahlan and Tahan [21] introduced the Generalized Numerical Inspection Fixture (GNIF) method which is based on the property that the shortest path (geodesic distance) between any two points on the surfaces does not change during an isometric displacement (distance preserving property of non-rigid parts) in spite of large displacement. Taking advantage of this property, the method looks for some correspondence between the part and the CAD model. The authors used Multidimensional Scaling in order to find a correspondence between two metric spaces (CAD model and scanned part). Then knowing some boundary conditions, finite element non-rigid registration (FENR) was executed. The geometric deviations between the displaced CAD model and the measurement data can be calculated after the FENR. Correspondence search is completely automatic. The GNIF dealt with a very general case of non-rigid inspection. In the absence of assembly conditions, the authors used the borders for FENR purposes. This situation may not conform to assembly conditions and real use state. Boundary conditions for the simulated displacements can be improved based on assembly conditions. The authors in [22] robustified the GNIF method by filtering out points that cause incoherent geodesic distances. The improved method is able to handle parts with missing data sets.

In contrast to the aforementioned methods, Abenhaim et al. [23] proposed the Iterative Displacement Inspection (IDI) algorithm that is not based on the FEA module. This method iteratively displaces the meshed CAD model until it matches the scanned data. The IDI algorithm is based on optimal step non-rigid ICP algorithms [24] which combine rigid and 
non-rigid registration methods. As well, a developed identification method distinguishes surface deviations from the part's displacement. This method principally displaces the mesh regarding its smoothness and prevents concealing surface defects and measurement noise during the matching process. Aidibe et al. [25] improved the identification module of the IDI algorithm by proposing the application of a maximum-normed residual test to automatically set the identification threshold. However, the IDI method has some drawbacks. Due to a lack of FE analysis, the method depends on identifying some flexibility parameters which are dependent on thickness. In addition, they use the same number of nodes in the two point clouds.

Aidibe and Tahan [12] presented an approach that combines the curvature properties of manufactured parts with the extreme value statistic test as an identification method for comparing two data sets and to recognize profile deviation. This approach was tested on simulated typical industrial sheet metal with satisfactory results in terms of error percentage in defect areas and in the estimated peak profile deviation. As the core of the algorithm is based on the Gaussian curvature comparison, application of the method is limited to relatively-flexible parts where small displacements are predictable. The authors in [26] proposed the IDB-ACPD method for optimization of the CPD algorithm in order to adapt it to the relatively-flexible parts problem, introducing two criteria: the stretch criterion between the nominal model and the aligned one, and the Euclidian distance criterion between the aligned nominal model and the scanned part.

\section{PROPOSED APPROACH}

In terms of registration problems, the literature tells us that the best approach seems to be to search for the correspondence between two data sets (in our case, the CAD model and the scanned data). As mentioned in the previous section, the GNIF method based on the isometric displacement [21] has some advantages that encourage us to use it to search for corresponding points between two data sets. In this paper, a new formulation of boundary conditions is defined, and the developed method is implemented on two industrial case studies with free-form surfaces.

\subsection{Proposed approach based on the improvement of displacement boundary conditions}

In the present method, we calculate the Cartesian coordinates of the matching points in both the data sets; then, we will improve the boundary conditions for the finite element analysis, by searching for the correspondents inside the predefined boundary areas.

The Generalized MDS method of non-rigid registration, applied in the GNIF approach, represents the corresponding points in the data sets based on the barycentric coordinate system [21] (Figure 2). But, to use these points for future purposes, their barycentric coordinates should be converted into Cartesian coordinates. Given a point with the barycentric coordinates $\left(\lambda_{1}, \lambda_{2}, \lambda_{3}\right.$, where $\left.\lambda_{1}+\lambda_{2}+\lambda_{3}=1\right)$ inside a triangle, and knowing the Cartesian coordinates of the vertices (the nodes of an element in the finite element mesh), the Cartesian coordinates can be obtained at the point through the following equations:

$$
\left\{\begin{array}{l}
x_{P}=\lambda_{1} \cdot x_{1}+\lambda_{2} \cdot x_{2}+\lambda_{3} \cdot x_{3} \\
y_{P}=\lambda_{1} \cdot y_{1}+\lambda_{2} \cdot y_{2}+\lambda_{3} \cdot y_{3} \\
z_{P}=\lambda_{1} \cdot z_{1}+\lambda_{2} \cdot z_{2}+\lambda_{3} \cdot z_{3}
\end{array}\right.
$$

By substituting $\lambda_{3}=1-\lambda_{1}-\lambda_{2}$ into the equations above:

$$
\left\{\begin{array}{l}
x_{P}=\lambda_{1} \cdot x_{1}+\lambda_{2} \cdot x_{2}+\left(1-\lambda_{1}-\lambda_{2}\right) \cdot x_{3} \\
y_{P}=\lambda_{1} \cdot y_{1}+\lambda_{2} \cdot y_{2}+\left(1-\lambda_{1}-\lambda_{2}\right) \cdot y_{3} \\
z_{P}=\lambda_{1} \cdot z_{1}+\lambda_{2} \cdot z_{2}+\left(1-\lambda_{1}-\lambda_{2}\right) \cdot z_{3}
\end{array}\right.
$$


Using Equations 2, the Cartesian coordinates of the corresponding points in each data set can be calculated.

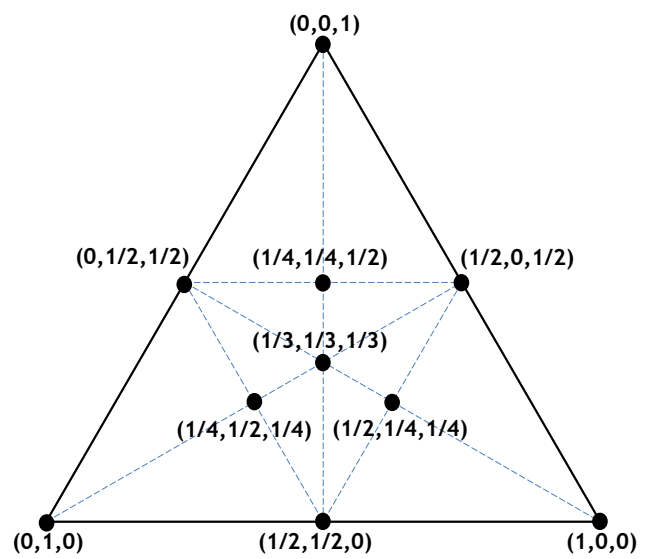

Figure 2: Barycentric coordinates $\left(\lambda_{1}, \lambda_{2}, \lambda_{3}\right)$ on an equilateral triangle

Figure 3 shows schematically the different steps of our approach. First, we put the scanned part surface $\left(S_{\text {Scan }}=p_{i}^{\prime}, i=1 \ldots m\right)$ close enough to the CAD surface $\left(S_{C A D}=p_{i}, i=1 \ldots n\right)$ (pre-alignment) to achieve a satisfactory result for rigid registration by ICP [8]. Then, the pre-aligned surface is rigidly registered to the CAD surface by the ICP algorithm. In this step, the GNIF method is used to find a set of correspondent pairs between the two surfaces:

$$
C_{C A D}=\left\{p_{k} \in S_{C A D} \mid k=1 \ldots q\right\} C_{S c a n}=\left\{p^{\prime}{ }_{k} \in S_{\text {Scan }} \mid k=1 \ldots q\right\}, \quad q \ll m, n
$$

To define a set of displacement boundary conditions for simulating the displacement from the CAD model to the rigidly registered scanned part surface, the constrained areas on the CAD model, such as fixation positions (e.g. hole) or contact surfaces (e.g. target datums) according to ASME Y14.5, are first recognized [20]. Then, the corresponding points (with the Cartesian coordinates) inside each constrained area (with the index of $j$ ), and consequently their correspondents in the scanned data, are identified among all the correspondents obtained by the GNIF method as follows:

$$
B_{j}=\left\{p_{t} \in C_{C A D} \mid t=1 \ldots s_{j} \ll q\right\}, B^{\prime}{ }_{j}=\left\{p_{t}^{\prime} \in C_{S c a n} \mid t=1 \ldots s_{j} \ll q\right\}
$$

Next, for each area and its corresponding area on the scanned surface, we define a centre of mass by fitting a plane through the identified corresponding points $\left(B_{j}, B_{j}^{\prime}\right)$. To register each pair of the identified correspondents in the two data sets by simulated displacement using finite element analysis, the displacement boundary conditions should be defined by local translation law [20]: (Figure 4)

- The centre of mass $\left(C_{m_{j}}\right)$ is translated to the corresponding centre of mass on the corresponding plane $\left(C_{m_{j}}^{\prime}\right)$ :

$$
\overrightarrow{\Delta r_{j}}=\left\{\begin{array}{l}
x_{c^{\prime}}-x_{c} \\
y_{c^{\prime}}-y_{c} \\
z_{c^{\prime}}-z_{c}
\end{array}\right\}_{j}
$$

Having defined the displacement boundary conditions, the finite element analysis is performed between the two data sets based on the simulated displacement approach. Using ANSYS $^{\circledR}$, the CAD model is displaced towards the scanned surface applying the defined boundary conditions. Finally, the profile deviations are identified based on the shortest 3D distance between each point of the scanned data and the displaced CAD surface $\left(\delta_{i}=\vec{\Delta} \cdot \vec{n}\right)$. 


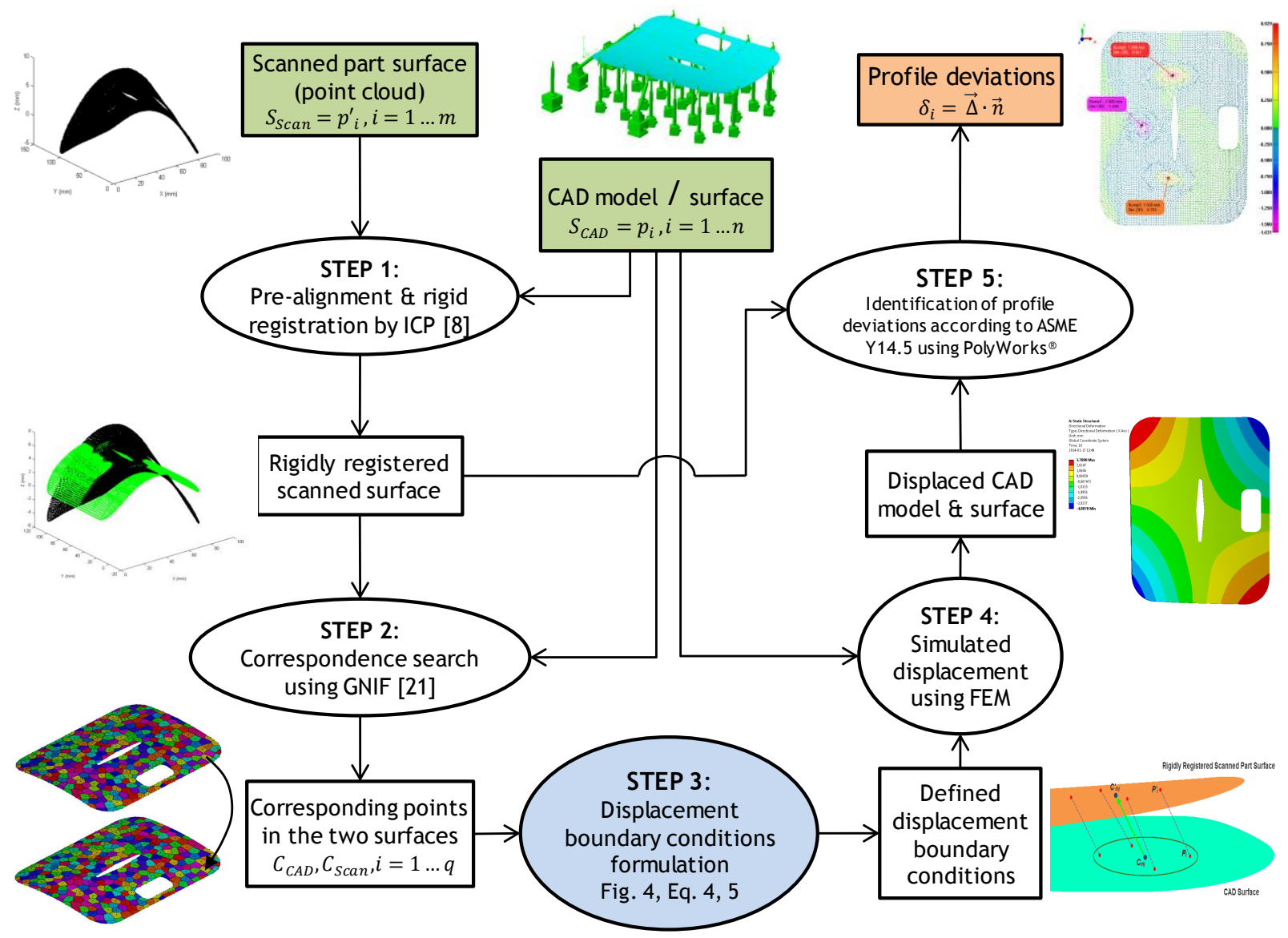

Figure 3: Flowchart of the proposed approach

Rigidly Registered Scanned Part Surface

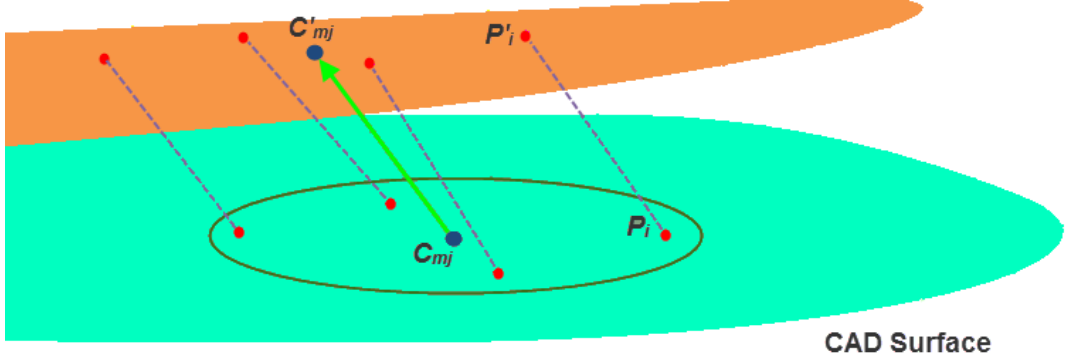

Figure 4: Definition of boundary conditions (step 3); correspondence points inside each constraint area and their correspondents on the scanned surface, centres of mass, and a displacement vector are illustrated (case A)

\section{CASE STUDIES}

We evaluated our approach on two industrial non-rigid part models from our aerospace industrial partner, Bombardier Aerospace. The parts are illustrated in Figure 5 . For each model, different virtual parts with different (but known) displacements and deviations (bumps) are simulated, and their point clouds are extracted. To simulate the parts, we applied two types of displacement (torsional or flexural), two types of defect area (small or big), and different amplitudes $(1,1.5$, or $2 \mathrm{~mm}$ ) on each model (A and B): A.S.F, A.S.T, A.B.F, A.B.T, B.S.F, B.S.T, B.B.F, B.B.T (case A or B, S: small defects, B: big defect, F: flexural displacement, $\mathrm{T}$ : torsional displacement). There is one defect in the cases with big area defects, and there are two or more defects with different amplitudes in the cases with small area defects. 
To evaluate repeatability of the approach, Gaussian measurement noise $N\left(0, \sigma_{\text {noise }}\right)$ was introduced three times on the case B.B.F where $\sigma_{\text {noise }}=0.02 \mathrm{~mm}$ that is a typical value of the measurement noise for a non-contact scanning device. Therefore, the proposed approach was applied on eleven (11) case studies.

In each case, first the pre-alignment and the rigid registration using the ICP algorithm are performed. Figure 6 shows the simulated parts after this step. Using the GNIF method, the correspondents between the CAD surface and the rigidly registered surface are recognized. (Figure 7)
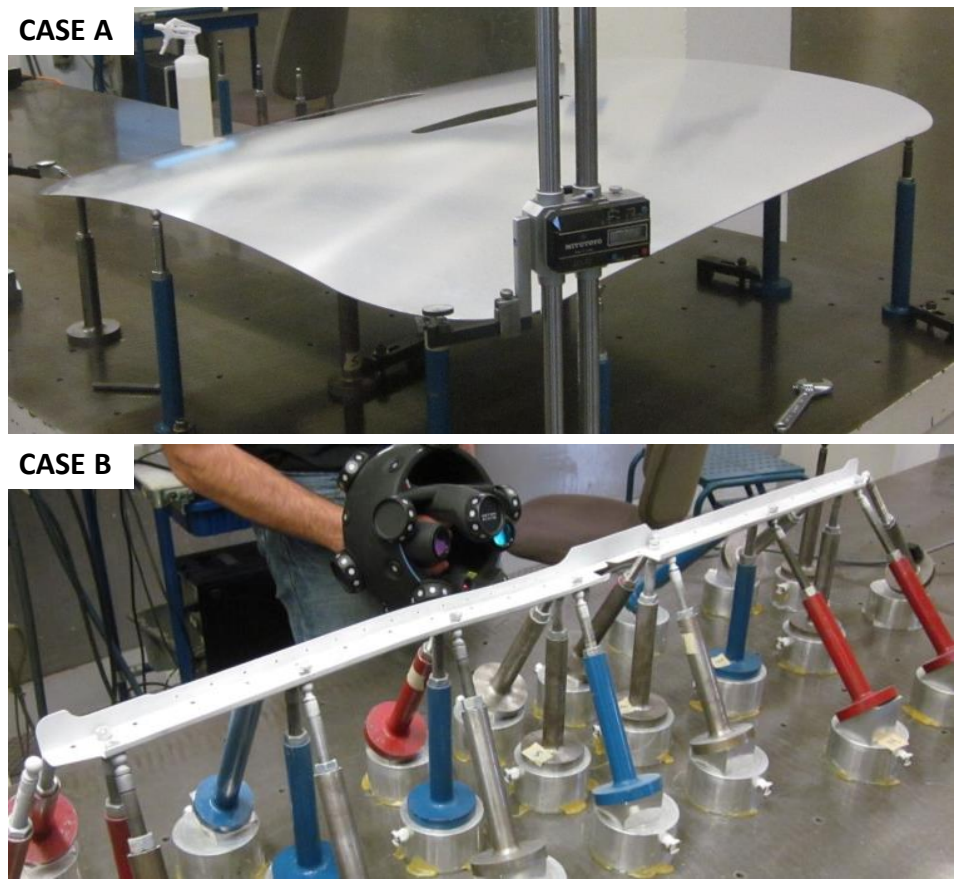

Figure 5: Non-rigid parts, Bombardier Aerospace Inc.

Small Area Defects

CASE A
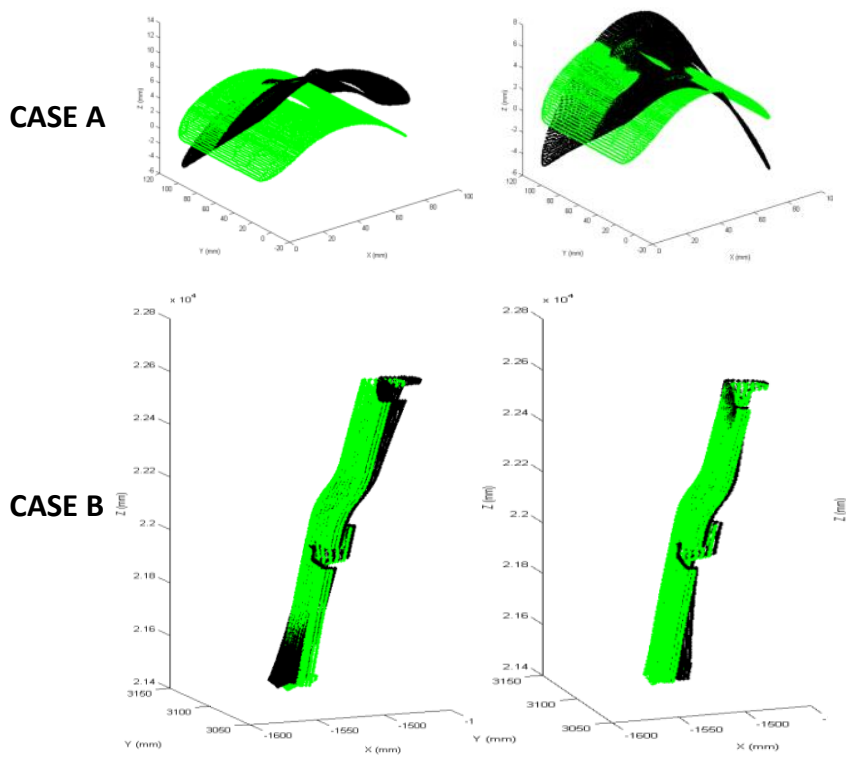

Big Area Defects

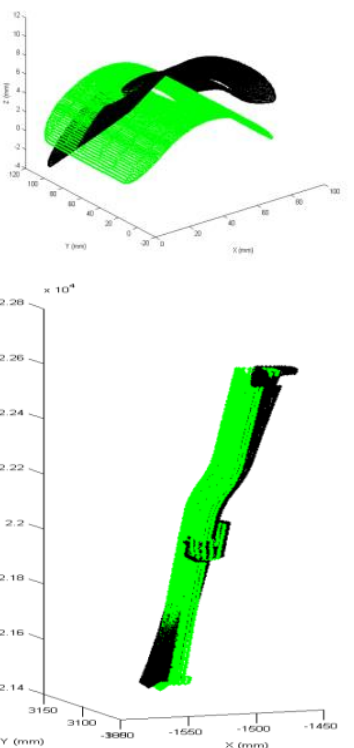

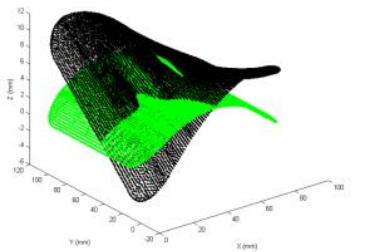

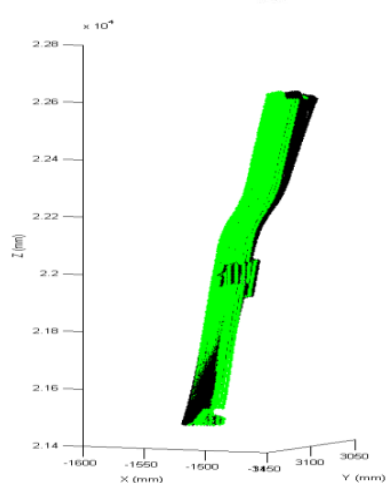

Figure 6: Simulated parts with different (but known) displacements and deviations, after pre-alignment and rigid registration (step 1) 

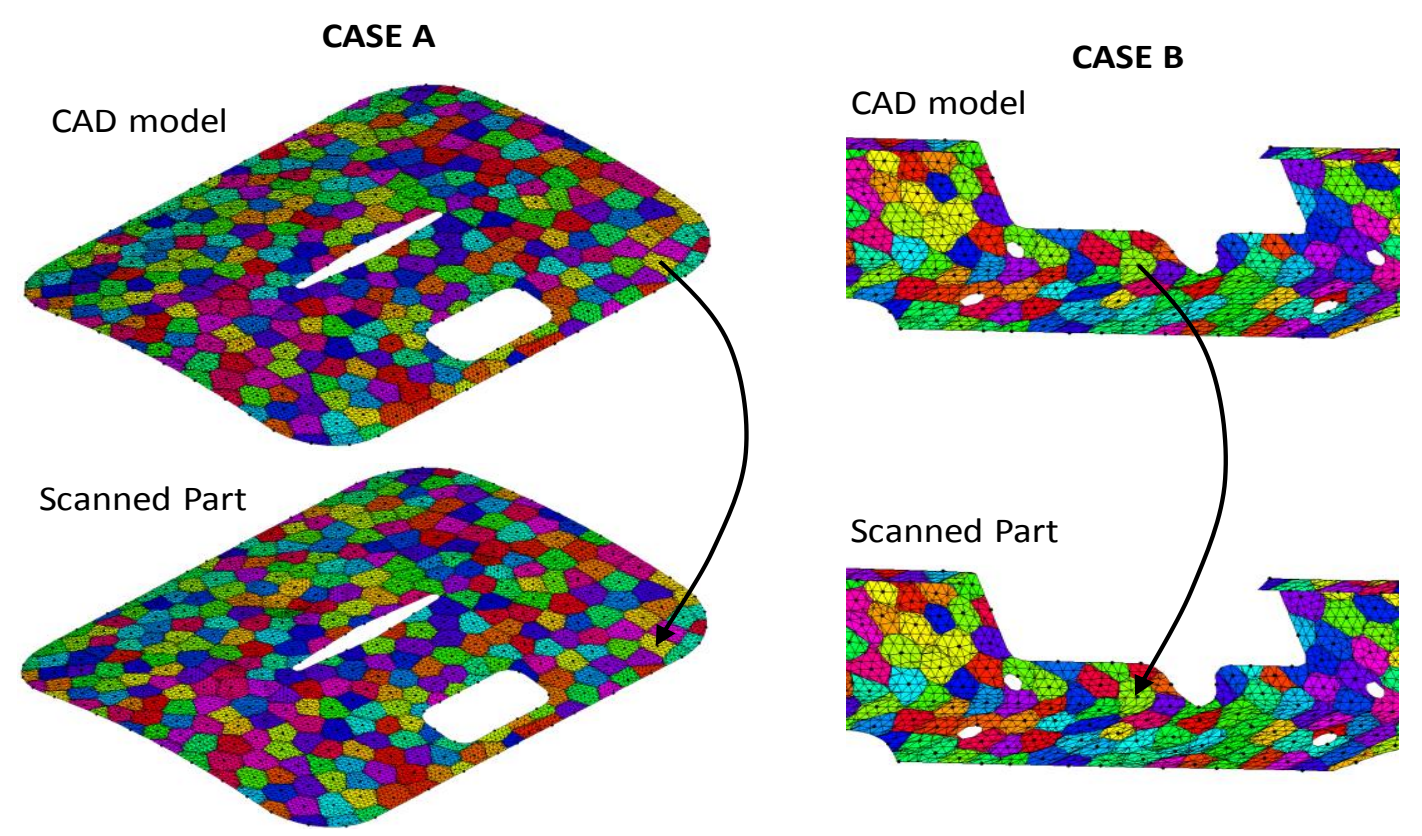

Figure 7: Correspondence search by GNIF (step 2) - example: cases A.S.T (case A, small defects, torsional displacement) and B.S.T (case B, small defects, torsional displacement)

Knowing the constrained areas and the corresponding points, the boundary conditions are defined. Then using ANSYS ${ }^{\circledR}$, the CAD model is displaced to the rigidly registered scanned surface for the FE non-rigid registration applying the linear elastic FEA method. The material is aluminum alloy 7050-T7451. Figure 8 shows the displacement results by FEM and the resulting displaced CAD surface.
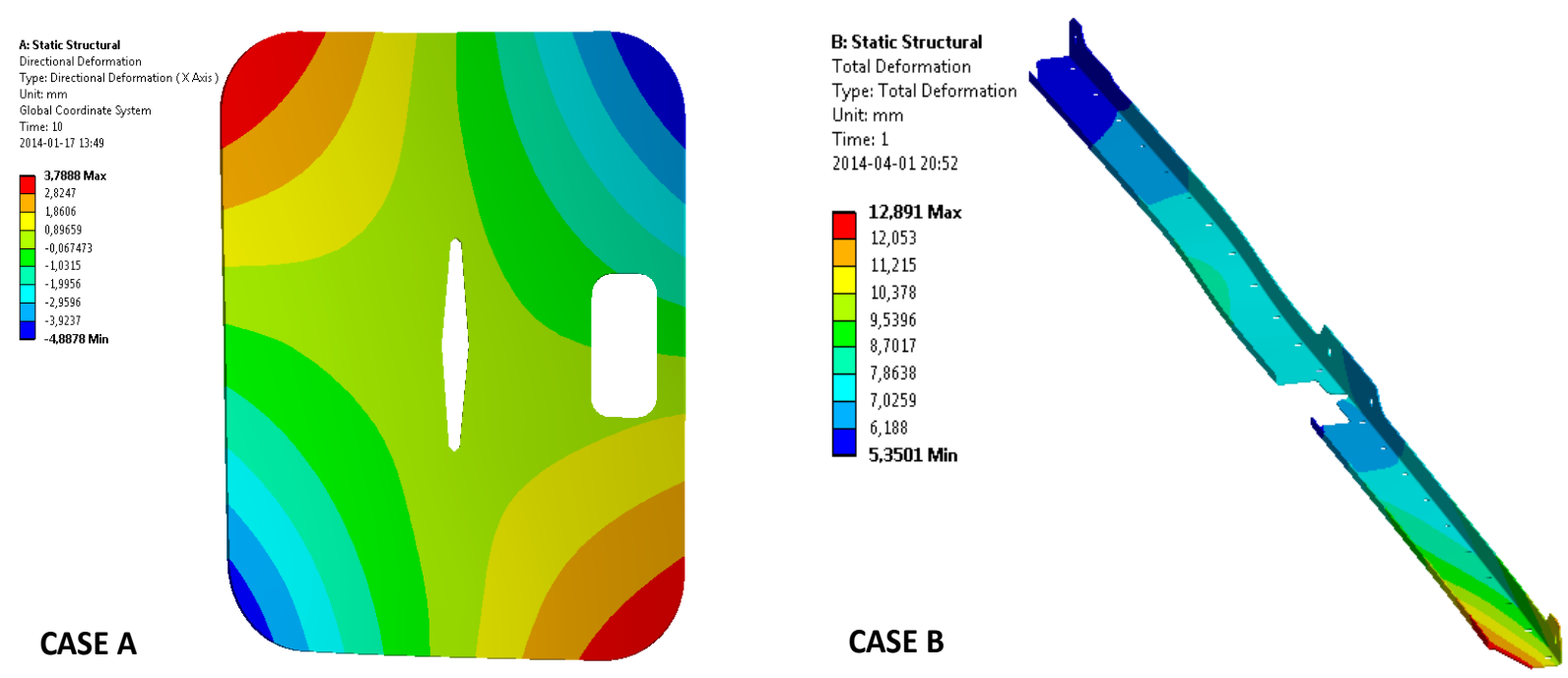

Figure 8: Displacement compensation by finite element analysis with defined boundary conditions between the CAD model and the rigidly registered surface, in ANSYS ${ }^{\circledR}$ (step 4) - example: cases A.S.T and B.S.T

Comparing the displaced CAD surface and the rigidly registered scanned surface, the known deviations are recognized, using PolyWorks ${ }^{\circledR}$. Table 2 represents a summary of the amplitude results in each defect compared between the nominal (simulated) amplitude and the detected (calculated) amplitude. The displacements are, on average, about $10 \mathrm{~mm}$. These values, as well as defect positions and areas, are illustrated in Figure 9 (Case A), Figure 10 (Case B) and Figure 11 (Case B.B.F with Gaussian measurement noise) using the inspection color maps in PolyWorks ${ }^{\circledR}$. 
Table 2: Results of defect's amplitude

\begin{tabular}{|c|c|c|c|c|c|c|c|c|}
\hline \multicolumn{3}{|c|}{ Case studies } & \multicolumn{3}{|c|}{ Case A } & \multicolumn{3}{|c|}{ Case B } \\
\hline $\begin{array}{l}\text { Type of } \\
\text { Defects }\end{array}$ & $\begin{array}{l}\text { Displacement } \\
\text { Type }\end{array}$ & $\begin{array}{l}\text { Defect } \\
\text { number }\end{array}$ & $\begin{array}{l}\text { Nominal } \\
\text { Amplitude } \\
(\mathrm{mm})\end{array}$ & $\begin{array}{l}\text { Detected } \\
\text { Amplitude } \\
(\mathrm{mm})\end{array}$ & $\begin{array}{l}\text { Error } \\
(\%)^{*}\end{array}$ & $\begin{array}{l}\text { Nominal } \\
\text { Amplitude } \\
\text { (mm) }\end{array}$ & $\begin{array}{l}\text { Detected } \\
\text { Amplitude } \\
(\mathrm{mm})\end{array}$ & $\begin{array}{l}\text { Error } \\
(\%)^{*}\end{array}$ \\
\hline \multirow{8}{*}{$\begin{array}{l}\text { Small } \\
\text { Area }\end{array}$} & \multirow{4}{*}{ Flexural } & 1 & 1.500 & 1.562 & 4.1 & 1.500 & 1.286 & 14.3 \\
\hline & & 2 & 1.000 & 0.756 & 24.4 & 2.000 & 1.770 & 11.5 \\
\hline & & 3 & 1.000 & 0.926 & 7.4 & 2.000 & 1.993 & 0.3 \\
\hline & & 4 & - & - & - & 1.000 & 0.780 & 22.0 \\
\hline & \multirow{4}{*}{ Torsional } & 1 & 1.500 & 1.444 & 3.7 & 1.500 & 1.360 & 9.3 \\
\hline & & 2 & 1.000 & 0.921 & 7.9 & 2.000 & 2.080 & 4.0 \\
\hline & & 3 & 1.000 & 0.742 & 25.8 & 2.000 & 1.773 & 11.3 \\
\hline & & 4 & - & - & - & 1.000 & 0.908 & 9.2 \\
\hline \multirow{5}{*}{$\begin{array}{c}\text { Big } \\
\text { Area }\end{array}$} & Torsional & 1 & 1.500 & 1.148 & 23.5 & 1.000 & 0.982 & 1.8 \\
\hline & Flexural & 1 & 1.500 & 1.228 & 18.1 & 1.000 & 1.113 & 11.3 \\
\hline & $\begin{array}{c}\text { Flexural + } \\
N_{1}\left(0, \sigma_{\text {noise }}\right)^{* *}\end{array}$ & 1 & - & - & - & 1.000 & 1.126 & 12.6 \\
\hline & $\begin{array}{c}\text { Flexural + } \\
N_{2}\left(0, \sigma_{\text {noise }}\right)\end{array}$ & 1 & - & - & - & 1.000 & 0.917 & 8.3 \\
\hline & $\begin{array}{c}\text { Flexural + } \\
N_{3}\left(0, \sigma_{\text {noise }}\right)\end{array}$ & 1 & - & - & - & 1.000 & 0.942 & 5.8 \\
\hline
\end{tabular}

* Error percentage in the result of defect amplitude

${ }^{* *} \sigma_{\text {noise }}=0.02 \mathrm{~mm}$ 


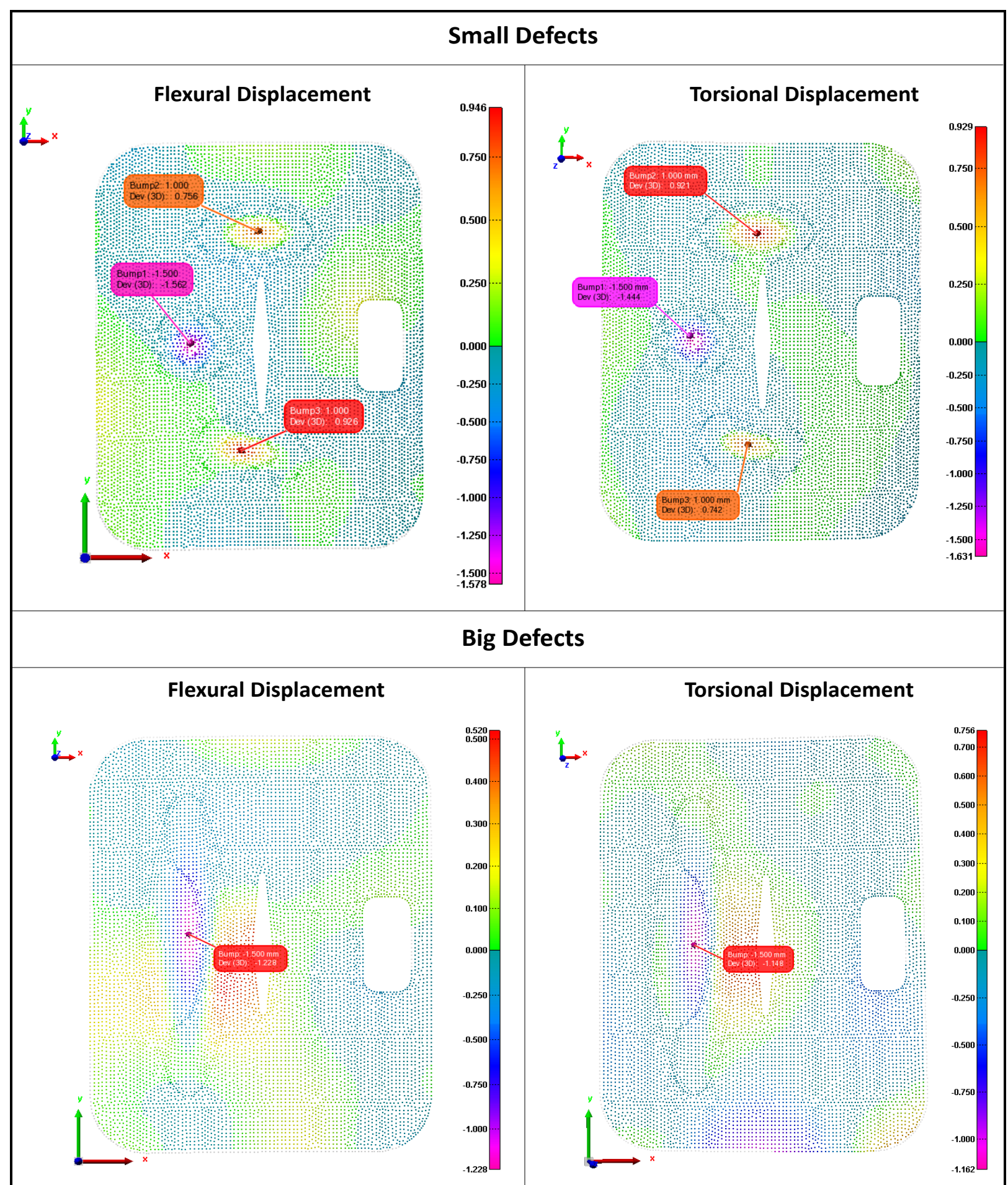

Figure 9: Defect amplitudes (mm), positions, and areas, using inspection color map - case A 


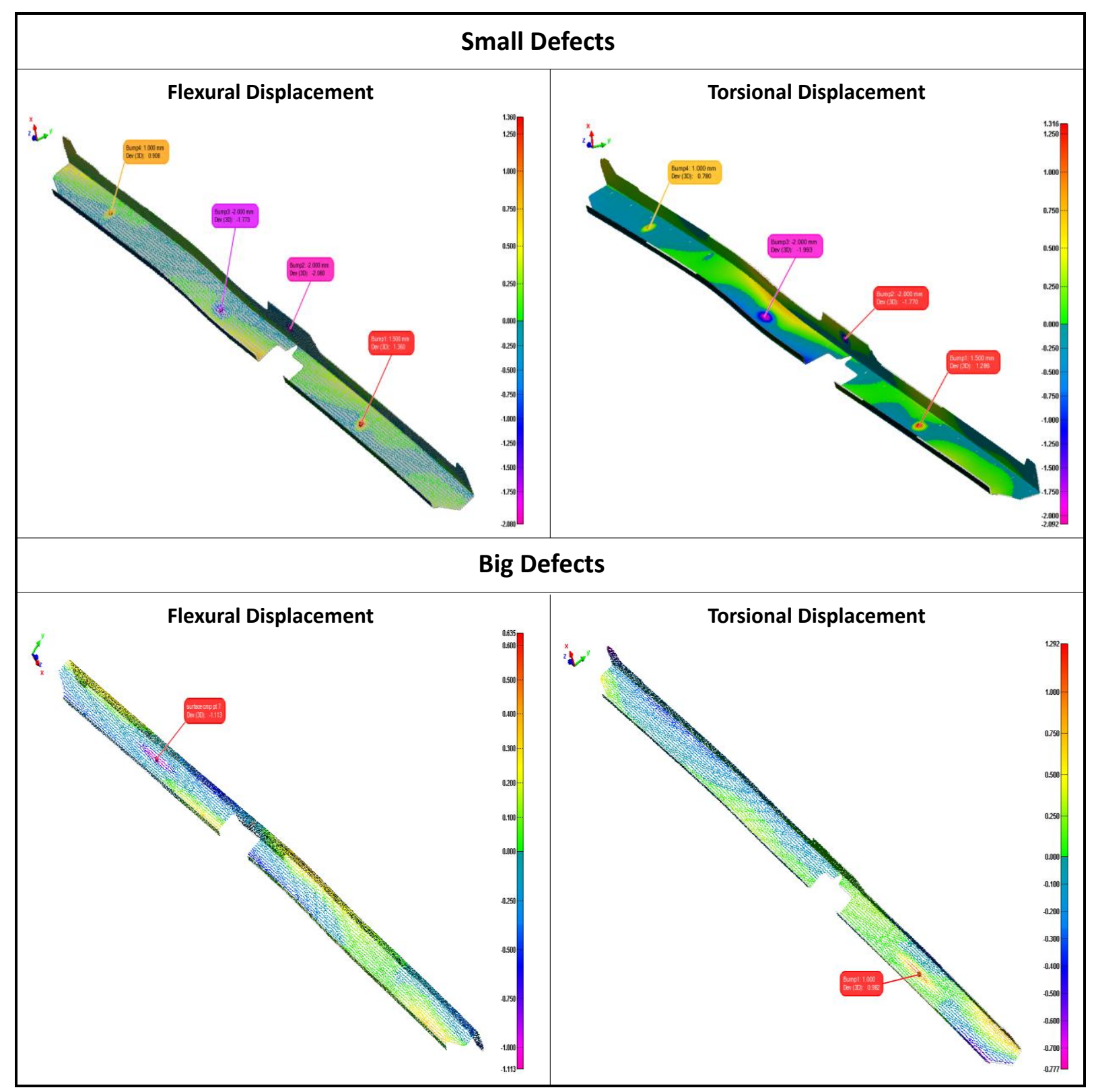

Figure 10: Defect amplitudes ( $\mathrm{mm})$, positions, and areas, using inspection color map case $B$ 


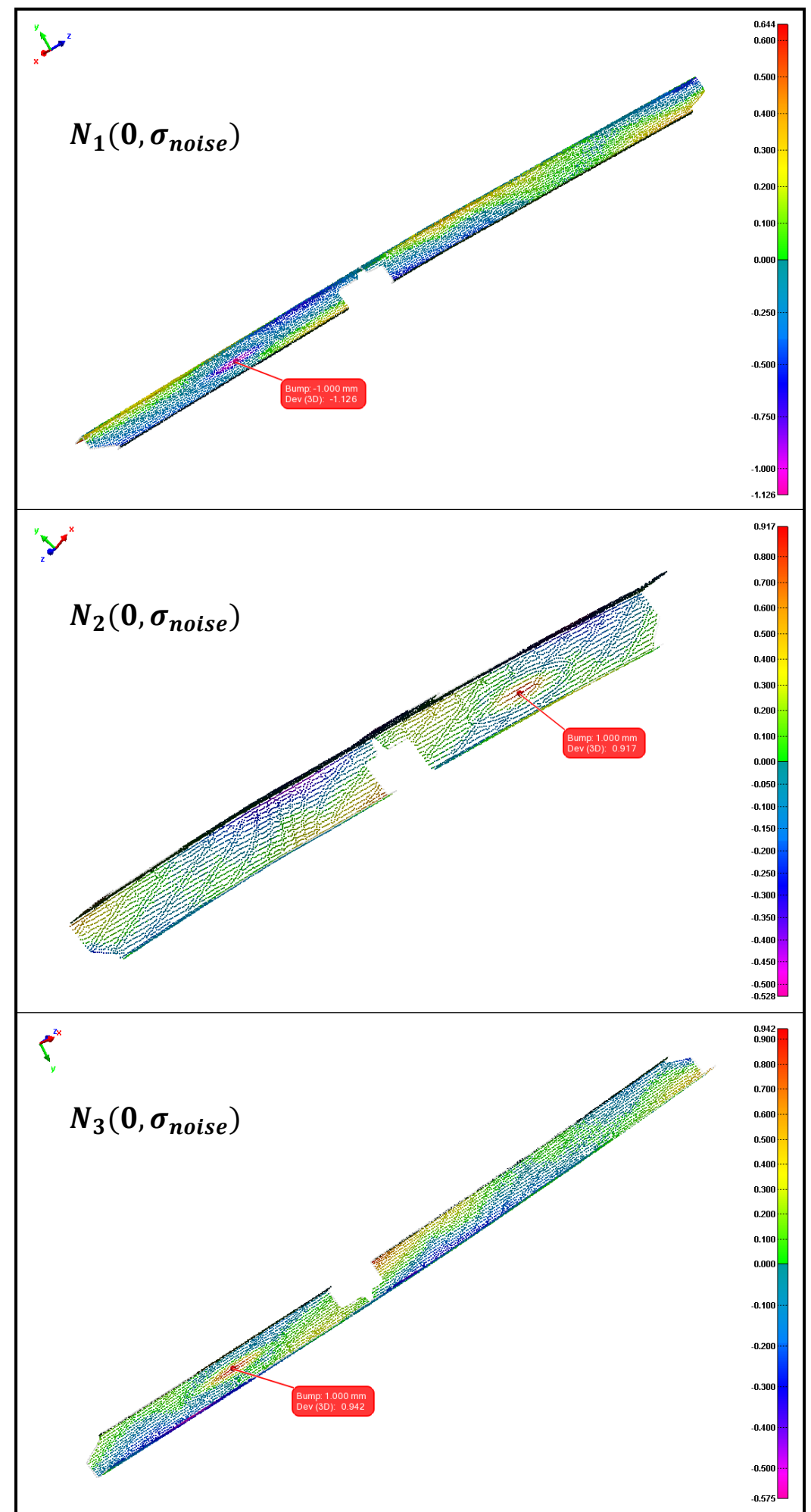

Figure 11: Defect amplitudes $(\mathrm{mm})$, positions, and areas, using inspection color map case B.B.F with Gaussian measurement noise $N\left(0, \sigma_{\text {noise }}\right), \sigma_{\text {noise }}=0.02 \mathrm{~mm}$

By improving the definition of boundary conditions, the error percentage generally decreases. A precise and complete definition of boundary conditions eventuates in precise results. Also, the accuracy of the correspondence searching method (GNIF in our paper) definitely affects the results. In a case where a defect (like the defect in the cases A.B.F and A.B.T) is located on a boundary area (a contact surface for example), its position and amplitude can be detected and calculated by studying and filtering the neighborhood area. 


\section{CONCLUSION}

In this paper, a technique for the profile inspection of flexible parts was developed to eliminate the need for specialized inspection fixtures. This approach was studied and evaluated on two industrial non-rigid part models from our industrial partner, Bombardier Aerospace Inc. To compare a point cloud (extracted from a simulated part containing known displacement and deviations) with the CAD model, a pre-alignment and a rigid registration (using the ICP method) were performed first. Next, applying the GNIF method, correspondents between the two data sets were found. Knowing the constrained areas such as contact surfaces and fixation areas on the CAD model, planes were fitted through the points inside each area as well as their correspondents on the scanned data. Then, the displacement boundary conditions were completely defined by local translation laws for finite element simulation. The deviation amplitudes, areas, and positions were identified comparing the scanned data with the displaced CAD model. In this paper, the improved method was applied on two industrial case studies with free-form complex surfaces. A definition of boundary conditions, and consequently, an identification of deviations were improved using our approach. If the boundary conditions are completely and exactly defined, more precise results will inevitably be obtained. Repeatability of the proposed approach was evaluated by introducing Gaussian measurement noise on a case. In the future work, repeatability of the approach as well as the detection of defect areas will be studied precisely. Our research advances to implement this approach on real point clouds acquired from part surfaces in order to improve the definition of, and to consider different kinds of, boundary conditions.

\section{ACKNOWLEDGMENTS}

The authors would like to thank the National Sciences and Engineering Research Council (NSERC) and our industrial partners for their support and financial contribution.

\section{REFERENCES}

1. Gao, J., N. Gindy, and X. Chen, An automated GD\&T inspection system based on noncontact 3D digitization. International journal of production research, 2006. 44(1): $p$. 117-134.

2. $\mathrm{Li}, \mathrm{Y}$. and P. Gu, Inspection of free-form shaped parts. Robotics and ComputerIntegrated Manufacturing, 2005. 21(4-5): p. 421-430.

3. ASME Y14.5-2009, in Dimensioning and tolerancing. 2009, The American Society of Mechanical Engineers National Standard: The American Society of Mechanical Engineers, New York.

4. ISO 1101:2004, in Geometrical product specifications (GPS)-geometrical tolerancing-tolerances of form, orientation, location and run-out. 2004, International Organization for Standardization (ISO): Geneva.

5. ISO 10579:2010, in Geometrical product specifications (GPS)-dimensioning and tolerancing-non-rigid parts. 2010, International Organization for Standardization (ISO): Geneva.

6. Abenhaim, G.N., et al., A novel approach for the inspection of flexible parts without the use of special fixtures. Journal of Manufacturing Science and Engineering, 2011. 133(1).

7. $\mathrm{Li}, \mathrm{Y}$. and P. Gu, Free-form surface inspection techniques state of the art review. Computer-Aided Design, 2004. 36(13): p. 1395-1417.

8. Besl, P.J. and N.D. McKay, A method for registration of 3-D shapes. IEEE Transactions on pattern analysis and machine intelligence, 1992. 14(2): p. 239-256. 
9. Borg, I. and P.J.F. Groenen, Modern multidimensional scaling: Theory and applications. 2005: Springer Verlag.

10. Myronenko, A. and S. Xubo, Point Set Registration: Coherent Point Drift. Pattern Analysis and Machine Intelligence, IEEE Transactions on, 2010. 32(12): p. 2262-2275.

11. Abenhaim, G.N., A. Desrochers, and S.A. Tahan, Nonrigid parts' specification and inspection methods: notions, challenges, and recent advancements. The International Journal of Advanced Manufacturing Technology, 2012. 63(5-8): p. 741752.

12. Aidibe, A. and A. Tahan, The inspection of deformable bodies using curvature estimation and Thompson-Biweight test. The International Journal of Advanced Manufacturing Technology, 2014: p. 1-15.

13. Ascione, R. and W. Polini, Measurement of nonrigid freeform surfaces by coordinate measuring machine. The International Journal of Advanced Manufacturing Technology, 2010. 51(9-12): p. 1055-1067.

14. Weckenmann, A. and A. Gabbia, Testing formed sheet metal parts using fringe projection and evaluation by virtual distortion compensation, in Fringe 2005, W. Osten, Editor. 2006, Springer Berlin Heidelberg. p. 539-546.

15. Weckenmann, A. and J. Weickmann, Optical inspection of formed sheet metal parts applying fringe projection systems and virtual fixation. Metrology and Measurement Systems, 2006. 13(4): p. 321-334.

16. Weckenmann, A., J. Weickmann, and N. Petrovic. Shortening of Inspection Processes by Virtual Reverse Deformation. in Proceedings of the CIRP 4th International Conference and Exhibition on Machines and Design and Production of Dies and Molds. 2007. Cesme, Izmir, Turkey.

17. Jaramillo, A.E., P. Boulanger, and F. Prieto. On-line 3-D inspection of deformable parts using FEM trained radial basis functions. in IEEE 12th International Conference on Computer Vision Workshops (ICCV Workshops). 2009. Kyoto, Japan: IEEE.

18. Jaramillo, A.E., P. Boulanger, and F. Prieto, On-line 3-D system for the inspection of deformable parts. The International Journal of Advanced Manufacturing Technology, 2011. 57(9-12): p. 1053-1063.

19. Jaramillo, A., F. Prieto, and P. Boulanger, Fast dimensional inspection of deformable parts from partial views. Computers in Industry, 2013(0).

20. Gentilini, I. and K. Shimada, Predicting and evaluating the post-assembly shape of thin-walled components via 3D laser digitization and FEA simulation of the assembly process. Computer-Aided Design, 2011. 43(3): p. 316-328.

21. Radvar-Esfahlan, H. and S.A. Tahan, Nonrigid geometric metrology using generalized numerical inspection fixtures. Precision Engineering, 2012. 36(1): p. 1-9.

22. Radvar-Esfahlan, H. and S.-A. Tahan, Robust generalized numerical inspection fixture for the metrology of compliant mechanical parts. The International Journal of Advanced Manufacturing Technology, 2014. 70(5-8): p. 1101-1112.

23. Abenhaim, G.N., et al., A Novel Approach for the Inspection of Flexible Parts Without the Use of Special Fixtures. Journal of Manufacturing Science and Engineering, 2011. 133(1): p. 011009-11.

24. Amberg, B., S. Romdhani, and T. Vetter. Optimal Step Nonrigid ICP Algorithms for Surface Registration. in IEEE Conference on Computer Vision and Pattern Recognition. 2007. Minneapolis, MN, USA. 
25. Aidibe, A., A. Tahan, and G. Abenhaim, Distinguishing profile deviations from a part's deformation using the maximum normed residual test. WSEAS Transactions on Applied and Theoretical Mechanics, 2012. 7(1): p. 18-28.

26. Aidibe, A. and A. Tahan, Adapting the coherent point drift algorithm to the fixtureless dimensional inspection of compliant parts. The International Journal of Advanced Manufacturing Technology, 2015: p. 1-11. 